\title{
Capillary waves on a falling film
}

\author{
Guangzhao Zhou $\odot^{*}$ \\ Department of Mechanical Engineering, University of Houston, 4726 Calhoun Road, \\ Houston, Texas 77204-4006, USA \\ Andrea Prosperetti ${ }^{\dagger}$ \\ Department of Mechanical Engineering, University of Houston, 4726 Calhoun Road, \\ Houston, Texas 77204-4006, USA \\ and Faculty of Science and Technology and J.M. Burgers Centre for Fluid Dynamics, \\ University of Twente, P.O. Box 217, 7500 AE Enschede, The Netherlands
}

(Received 6 November 2019; accepted 27 October 2020;

published 24 November 2020)

\begin{abstract}
This paper shows that the wavelength and rate of spatial attenuation of the small ripples accompanying the large waves that form on a liquid film flowing down an inclined plane are dictated by the requirement that their velocity equal the velocity of the large wave. For this purpose numerical simulations of film flow are carried out and validated by comparison with published work. The wavelength and rate of spatial attenuation of the computed capillary waves are shown to equal, to an excellent approximation, the corresponding quantities obtained from an approximate form of the Orr-Sommerfeld equation in which the phase velocity is set equal to the velocity of the large waves. Thus the overall structure of the falling film waves is similar to that encountered in many other problems exhibiting a wave-hierarchical structure, such as waves in dusty or reacting gases, hydraulic jumps, undular bores, and others.
\end{abstract}

DOI: 10.1103/PhysRevFluids.5.114005

\section{INTRODUCTION}

There are many wave phenomena which exhibit a hierarchical structure in the sense that the main wave is accompanied by smaller waves, the characteristics and location of which are determined by the requirement that their phase velocity matches that of the main wave and their group velocity be such that energy be transported away from the main wave (see, e.g., Ref. [1]). Well-known examples occur, e.g., in the free-surface flow disturbed by a submerged body (see, e.g., Refs. [1,2]) or other agents (see, e.g., Ref. [3]), shock waves in reacting or relaxing gases (see, e.g., Ref. [4]), or in bubbly liquids (see, e.g., Ref. [5]), traffic flow (see, e.g., Ref. [6]), and many others.

In this paper we identify yet another example of this general pattern in the flow of a liquid film down an inclined plane. The wave pattern that develops in this flow, either naturally or under the action of periodic forcing at the inlet, is characterized by a train of large waves, each one accompanied by much shorter and smaller-amplitude ripples (see examples in Figs. 1, 5, and 6). We show that the wavelength and rate of spatial attenuation of these capillary waves are dictated precisely by the requirement that they propagate at the same speed as the large waves to which they are, in a sense, enslaved. In the literature there is a brief reference to this aspect in Ref. [7], but the

\footnotetext{
*gzhou5@central.uh.edu

†aprosper@central.uh.edu
} 

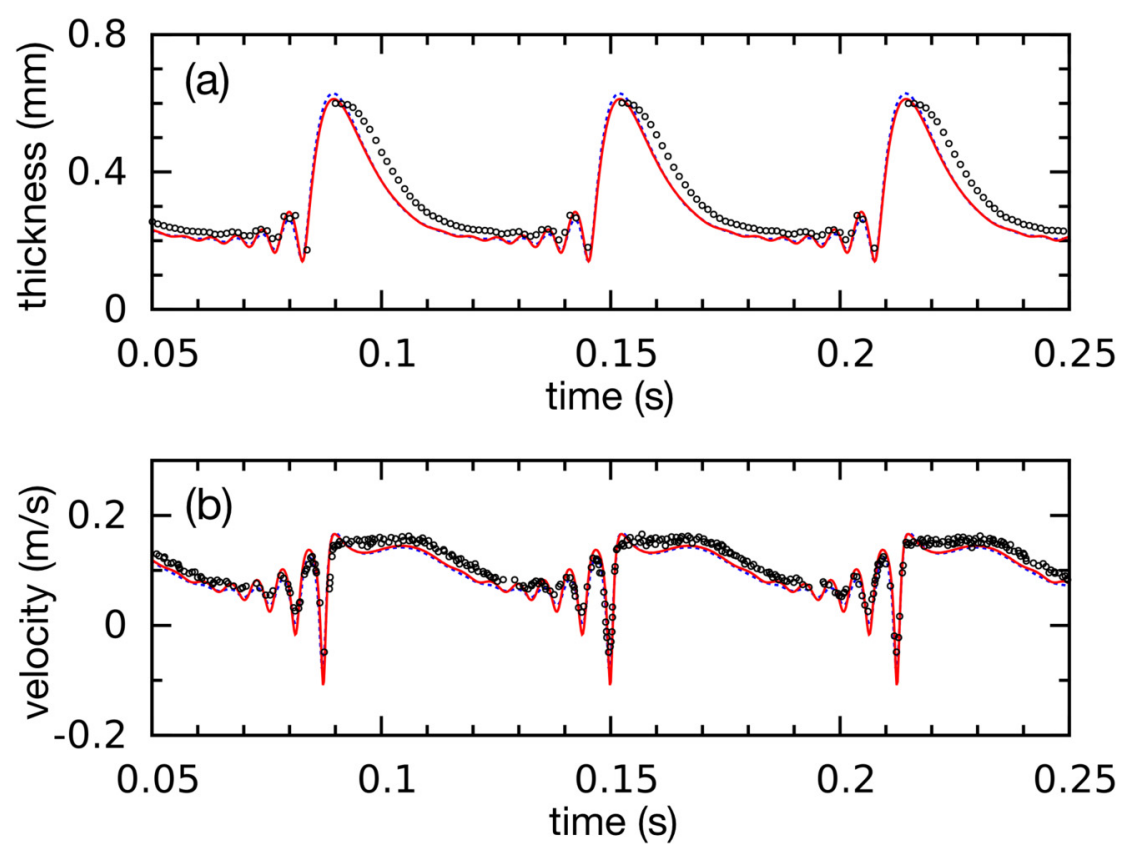

FIG. 1. The open circles show the measured film height (top) and streamwise velocity at a distance of $120 \mu \mathrm{m}$ from the wall (bottom) reported by [33]. The solid lines are the results of the present simulations, and the barely distinguishable dotted lines are the numerical results reported in [23]; see text for parameter values.

idea does not seem to have been followed up. A similar association of capillary ripples and much larger waves is encountered, e.g., near the crest of breaking gravity waves as shown, among others, in Refs. [8,9]. The large primary wave and the smaller waves that accompany it are components of a single structure that can be related to a homoclinic orbit in the phase space of the system (see, e.g., Refs. $[10,11])$. Nevertheless, the separation of scales between these two components makes it possible to study them separately.

It has been known at least since the seminal work of P.L. Kapitza [12] that the film surface ceases to remain plane as soon as the Reynolds number exceeds a critical value, which becomes smaller and smaller as the inclination angle increases, until it vanishes for a vertical wall. The understanding of the complex wave structure that develops as a result of this instability has proven challenging for theory (see, e.g., Refs. [7,10,11,13-15]) as well as computation (see, e.g., Refs. [16-18]). At the same time, it is this very wave structure that makes film flow scientifically interesting and endows it with a much greater ability to transfer mass and heat compared to a flat film, thus providing further stimulus for its study.

For obvious reasons much of previous work has focused on the large waves. A detailed focus on the mechanics of the small viscous ripples is a relatively recent development, both generally (see, e.g., Ref. [19]) and in the specific context of film flow. References $[15,17,20]$ have argued that, in spite of their smaller amplitude, these ripples play an important role in affecting the interaction and separation between consecutive large waves. Specifically, the capillary waves exert a draining action on the large waves, which affects their speed of propagation [11] and, with it, wave merging and splitting. Furthermore, capillary waves appear to have a significant effect on the transfer of mass and energy through the film since the transfer coefficient associated with them is approximately inversely proportional to the film thickness $[21,22]$.

Reference [23] describes a detailed study of capillary waves focusing on the mechanism of their formation and their speed, wavelength, and attenuation. The author argues that these small 
capillary waves are quite different from conventional capillary waves and owe their formation and characteristics to inherently nonlinear phenomena. By an analysis of wave patterns computed by the present authors and others from the literature, the present paper shows that nonlinear effects, while present, are not the determining factor. A linear approximation is adequate to satisfactorily account for these small ripples, which actually bear a strong similarity to linear viscocapillary waves on a horizontal liquid film of finite depth.

In the next section we give a very abbreviated exposition of our numerical method, which is described in greater detail in a recent paper [24], followed in Sec. III by the solution of an approximate form of the Orr-Sommerfeld equation from which the dispersion relation of the capillary waves is obtained. In this step we are forced to take an unusual view of this equation, solving it for a prescribed real velocity of the wave. The results are then applied to the capillary waves on a falling film in Sec. IV, where proof of the hierarchical nature of film flow is proven. The Appendix focuses on the relationship of our treatment of the Orr-Sommerfeld equation with other, more common approaches.

\section{NUMERICAL SIMULATIONS}

The objective of this paper requires detailed information on wavelength, wave speed, attenuation, and other features of the wave system on a falling film. Since the literature contains only a small number of suitable examples, it has been necessary to carry out additional simulations by a method that we now briefly describe. A more extensive exposition is available in a recent paper [24].

As in previous studies [18,23,25-27], in our numerical work we take advantage of the experimentally confirmed fact that periodic trains of waves can be generated by a suitable excitation at the inlet (see, e.g., Refs. [28-30]). The periodicity permits us to focus on a single spatial period, keeping the total fluid volume (or mass) in the computational domain constant. This approach is denoted as "closed flow" according to the classification of [31]. Following an approach common in the literature (see, e.g., Refs. [11,13]), we limit ourselves to two space dimensions, $x$ along the flow direction and $z$ normal to the plate.

The full Navier-Stokes equations are solved by a standard projection method. No-slip conditions are applied at the solid wall $z=0$ while, at the free surface, we apply the kinematic condition, the conditions of vanishing tangential viscous stress, and the condition that the normal stress be balanced by surface tension. Dynamical effects on the gas side of the interface are disregarded. The results depend on the Reynolds and Kapitza numbers

$$
\operatorname{Re}=\frac{\bar{h} \bar{U}}{v}, \quad \mathrm{Ka}=\frac{\sigma / \rho}{(g \sin \theta)^{1 / 3} v^{4 / 3}} .
$$

In these expressions, $g, \sigma, \rho$, and $v$ are the acceleration of gravity, the surface tension coefficient, and the density and kinematic viscosity of the liquid; $\bar{h}$ is the mean film thickness; and $\bar{U}$ the mean velocity of a flat film with thickness $\bar{h}$ flowing down a plate inclined at an angle $\theta$ to the horizontal:

$$
\bar{U}=\frac{\bar{h}^{2} g \sin \theta}{3 v} .
$$

The simulations shown later fall into the ranges $346 \leqslant \mathrm{Ka} \leqslant 6752$ and $11.1 \leqslant \mathrm{Re} \leqslant 19.3$.

The numerical method is based on a finite-difference discretization with colocated variables except that, following Ref. [32], the $z$ velocity component which is staggered in the direction $z$ normal to the wall. In order to deal with the unknown position of the free surface, we use a stretched coordinate $\zeta$ defined by $\zeta=z / h(x, t)$, with $h(x, t)$ the local instantaneous film thickness. The mesh length in the $x$ direction is about 10 times larger than in the $\zeta$ direction. We typically use 300 and 35 meshes in the streamwise and wall-normal directions, respectively. Thus, on the basis of our own experience and of information in the literature (see, e.g., Refs. [17,23]) we are confident to have a good spatial resolution. The time step was conservatively set on the basis of standard stability conditions. 
The calculation is carried out in a fixed (laboratory) reference frame. It is started with an imposed two-dimensional sinusoidal wave with the same wavelength as the computational domain. The fluid velocity is initiated according to the local Nusselt profile adjusted to the local film height. The wave propagates and develops in time until its shape no longer changes. The wave speed is calculated by tracking the velocity of the wave crest in the computational domain. The code is able to perform three-dimensional simulations but, due to the two-dimensionality of the initial condition, spanwise perturbations do not develop in the present simulations.

We have validated the code by comparing with results in the literature. An example is shown in Fig. 1, where the results of our simulations are superimposed on those reported in Ref. [33]; the pertinent parameter values are $\mathrm{Re}=11.1, \mathrm{Ka}=509.5, \ell / \bar{h}=68.87, \theta=90^{\circ}$. The open circles are the film height (top) and streamwise velocity at a distance of $120 \mu \mathrm{m}$ from the wall (bottom) reported in the reference. The solid lines are our simulations, and the barely distinguishable dotted lines under it the numerical results of Ref. [23]. In order to show this comparison we have converted our results from space to time using the computed phase velocity. As can be seen, the two computations are virtually identical and both closely match the data. Additional comparisons of this type can be found in Ref. [24].

\section{VISCOUS CAPILLARY WAVES ON A FALLING FILM}

The objective of this paper is to show that the ripples that accompany the large waves on a falling film are capillary waves, the velocity of which is dictated by the requirement that they appear steady in the frame of reference moving with the large wave. If this statement is correct, in the linear approximation, the Orr-Sommerfeld equation for the film flow should have solutions in the form of small-wavelength ripples that appear steady when viewed in the rest frame of the main wave propagating with velocity $c$. The wavelength and rate of spatial attenuation of these ripples should match the numerical results shown in the previous and the next sections. In other words, we should find solutions proportional to $\exp [i k(x-c t)]$, with $k$ the complex wave number. Of course, $k$ will be dependent on $c$, as waves which appear stationary in one frame will not have this character in a different frame. It may be noted that, as posed, the question is different from standard stability problems in which $k$ is real for temporal stability while the angular frequency $\omega$ is real for spatial stability. We briefly address this point in the Appendix.

Solution of the full Orr-Sommerfeld equation is a matter of some complexity. For simplicity, we adopt a useful simplification introduced in Ref. [34]. The authors show that approximating the velocity profile in the film by the constant surface velocity gives results in good agreement with the exact theory, with the error rapidly decreasing as the Kapitza number increases. Without reproducing the details, which are available in several references (see, e.g., Refs. $[11,13,34,35]$ ), we solve the Orr-Sommerfeld equation in the form

$$
\varphi^{\prime \prime \prime \prime}-2 k^{2} \varphi^{\prime \prime}+k^{4} \varphi=-i \frac{k}{v}\left[\left(c-\frac{3}{2} U\right)\left(\varphi^{\prime \prime}-k^{2} \varphi\right)+U^{\prime \prime} \varphi\right] .
$$

The stream function $\psi$ has been written as $\psi(x, y)=e^{i k(x-c t)} \varphi(y)$, and $U$ is the Nusselt mean velocity of the film given by (2) with $\bar{h}$ replaced by the mean thickness $h$ of the liquid layer supporting the small waves. Furthermore, $U^{\prime \prime}=-3 U / h^{2}$. The no-slip condition at the plate requires that $\varphi(0)=\varphi^{\prime}(0)=0$. The dynamic condition at the film surface $y=h$ is

$$
\left(c-\frac{3}{2} U\right)\left[\varphi^{\prime \prime \prime}-3 k^{2} \varphi^{\prime}+i \frac{k}{v}\left(c-\frac{3}{2} U\right) \varphi^{\prime}\right]-i k \frac{(\sigma / \rho) k^{2}+g \cos \theta}{v} \varphi=0,
$$

and the kinematic condition at the film surface $y=h$ is

$$
\left(c-\frac{3}{2} U\right)\left(\varphi^{\prime \prime}+k^{2} \varphi\right)+U^{\prime \prime} \varphi=0 .
$$



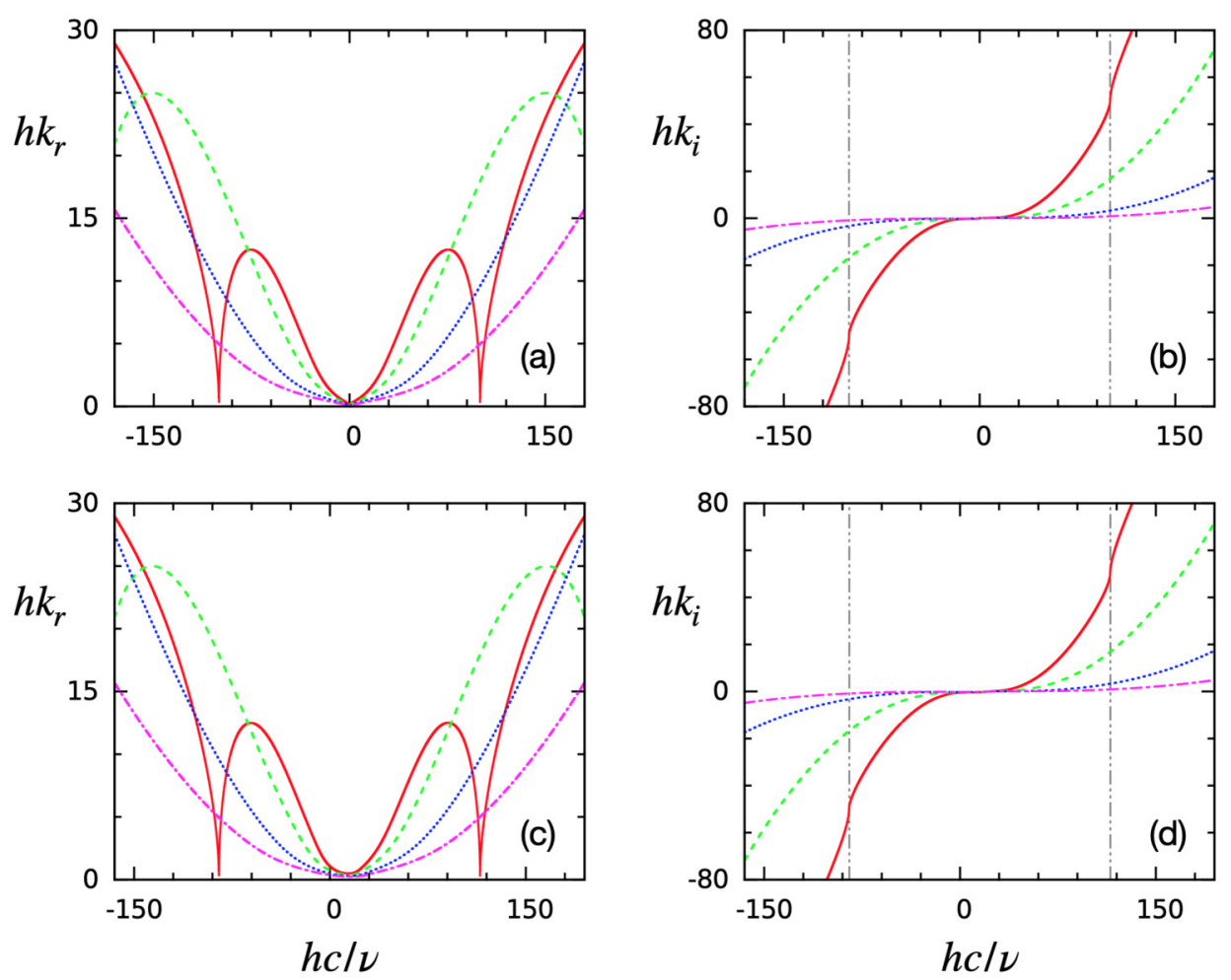

FIG. 2. Real (left) and imaginary parts of the dimensionless wave number $h k=h k_{r}+i h k_{i}$ as functions of the dimensionless wave velocity $h c / v$ for $\operatorname{Re}_{h}=0$ (upper pair of panels) and $\operatorname{Re}_{h}=10$. The lines are for $\Sigma=200$ (solid, red), 400 (dashed, green), 1000 (dotted, blue), and 2000 (dash-dotted, purple). Details near the minimum points of $k_{r}$ are shown in Figs. 3 and 4.

Since the approximate equation (3) has constant coefficients, its solution is the superposition of exponentials. We write

$$
\varphi=A \sin \beta_{+}(h-y)+B \cos \beta_{-}(h-y)+C \sin \beta_{+}(h-y)+D \cos \beta_{-}(h-y),
$$

in which

$$
\beta_{ \pm}=\left[-k^{2}+i \frac{k}{2 v} \hat{c} \pm i \sqrt{\frac{k^{2} \hat{c}^{2}}{4 v^{2}}+i \frac{k U^{\prime \prime}}{v}}\right]^{1 / 2}
$$

where we have written $\hat{c}=c-\frac{3}{2} U$ for convenience. Satisfaction of the boundary conditions leads to imposing the vanishing of the determinant

$$
\left|\begin{array}{cccc}
\sin \beta_{+} h & \sin \beta_{-} h & \cos \beta_{+} h & \cos \beta_{-} h \\
\beta_{+} \cos \beta_{+} h & \beta_{-} \cos \beta_{-} h & -\beta_{+} \sin \beta_{+} h & -\beta_{-} \sin \beta_{-} h \\
-\beta_{+} \gamma_{+} \hat{c} & -\beta_{-} \gamma_{-} \hat{c} & i\left[\frac{\sigma}{\rho \nu} k^{2}+\frac{g}{\nu} \cos \theta\right] k & i\left[\frac{\sigma}{\rho \nu} k^{2}+\frac{g}{v} \cos \theta\right] k \\
0 & 0 & \hat{c}\left(k^{2}-\beta_{+}^{2}\right)+U^{\prime \prime} & \hat{c}\left(k^{2}-\beta_{-}^{2}\right)+U^{\prime \prime}
\end{array}\right|=0 \text {, }
$$

in which $\gamma_{ \pm}=3 k^{2}+\beta_{ \pm}^{2}-i k \hat{c} / v$ (we note a misprint in the original Ref. [34] in which the term $k^{2}$ is erroneously preceded by a minus sign). In the present context, this is an equation for $k=k_{r}+i k_{i}$ as a function of the (real) velocity $c$ of the frame of reference in which the wave appears stationary. 

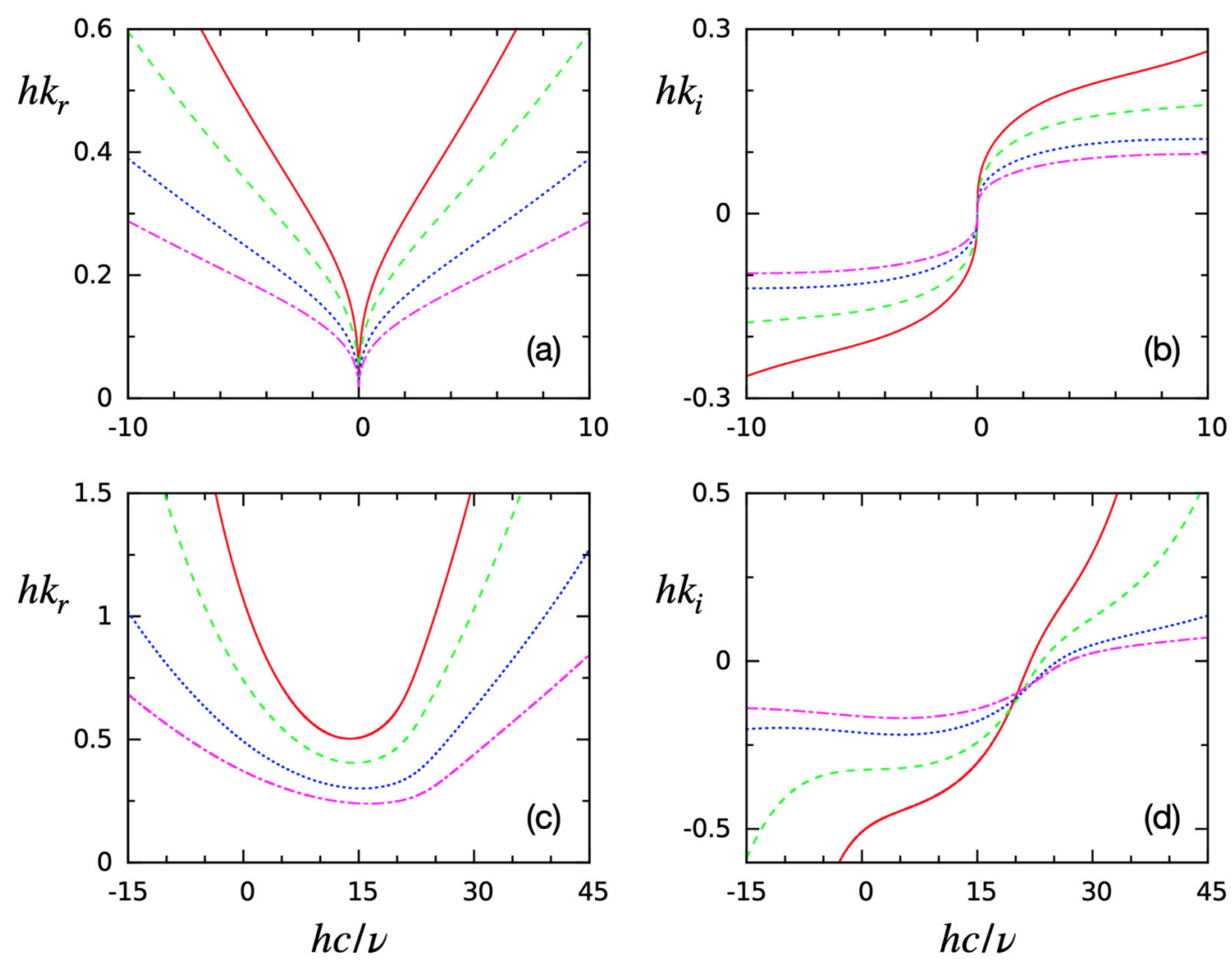

FIG. 3. Real (left) and imaginary parts of the dimensionless wave number $k h$ as functions of the dimensionless wave velocity $h c / v$ for small $c$. Here $\operatorname{Re}_{h}=0$ in the upper pair of panels and $\operatorname{Re}_{h}=10$ in the lower pair. The lines are for $\Sigma=200$ (solid, red), 400 (dashed, green), 1000 (dotted, blue), and 2000 (dash-dotted, purple).

We solve this equation by Muller's method [36]. Some results for the dimensionless wave number $k_{*}=h k$ in terms of the dimensionless wave velocity $c_{*}=h c / v$ are shown in Fig. 2 for a vertical plate with $\theta=\frac{\pi}{2}$. Since the mathematical statement of the problem (3) to (5) is invariant after complex conjugation followed by a sign change of the wave number, for every pair $\left(k_{r}, k_{i}\right)$ there is also a pair $\left(-k_{r}, k_{i}\right)$, which is not shown. The parameters are a Reynolds number $\operatorname{Re}_{h}$ and a dimensionless surface tension $\Sigma$, defined by

$$
\operatorname{Re}_{h}=\frac{U h}{v}, \quad \Sigma=\frac{\sigma h}{\rho v^{2}}
$$

Details of the results near $c=0$ are shown in Fig. 3 and near the other point where $k_{r}$ approaches zero for larger $|c|$ in Fig. 4.

For $\operatorname{Re}_{h}=0$ the curves are symmetric about $c=0$, as could have been expected. This result is different from the dispersion relation for capillary waves over a horizontal plate because, in that case, $k$ is taken to be real while it is complex here (see the Appendix). From the detail in Fig. 3(a), it is seen that $k_{r}$ vanishes and has a cusp at $c=0$, at which point, from Fig. 3(b), $k_{i}$ has a vertical tangent. Equation (A2) shows then that the group velocity, however defined, vanishes at this point. The cusp is regularized for $\operatorname{Re}_{h}=10$ [Fig. 3(c)] and, at the local minimum of $k_{r}$, the term $d c / d k_{r}$ gives a divergent contribution to the group velocity. 

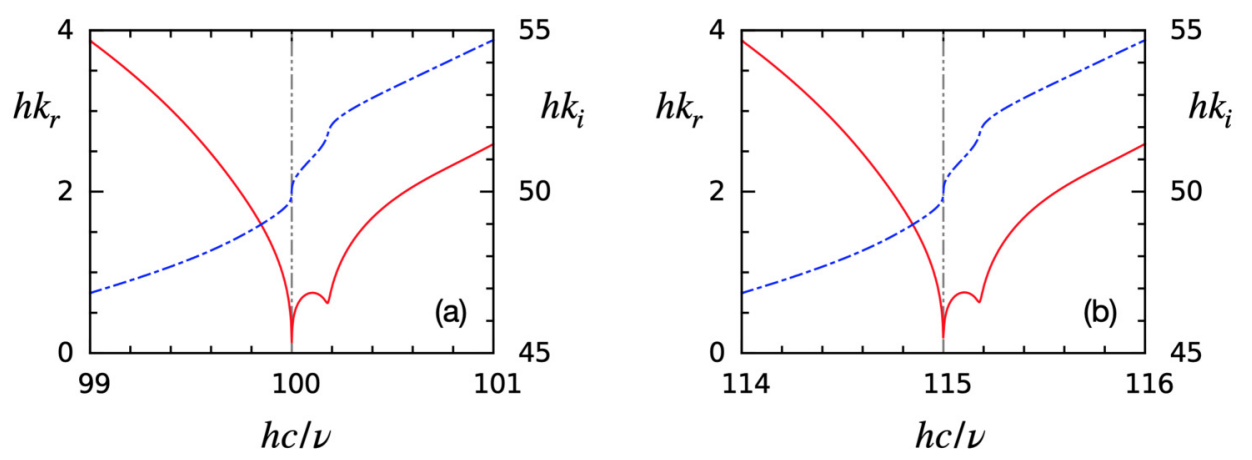

FIG. 4. Real (solid lines, red) and imaginary (dashed lines, blue) parts of the dimensionless wave number $k h$ as functions of the dimensionless wave velocity $h c / v$ in the neighborhood of the second minimum of $k_{r}$ for $\Sigma=200$. The left panel is for $\operatorname{Re}_{h}=0$ and the right one for $\operatorname{Re}_{h}=10$. The scales for $k_{r}$ and $k_{i}$ are on the left and right vertical axes, respectively.

With our choice of axes, the film flows in the direction of increasing $x$, which is the same as the direction of propagation of the waves if $c>0$. Thus for $c>0$, a positive value of $k_{i}$ implies that the amplitude of the waves decreases in the direction of propagation (which is the same as that of the flow). Conversely, a negative value of $k_{i}$ for $c<0$ corresponds to waves damped in the direction of propagation (which, in this case, is opposite that of the flow). Therefore the two situations are not substantially different from each other. However, $k_{i}<0$ for $c>0$ describes waves the amplitude of which increases in the direction of propagation. It can be seen in Fig. 3(d) that, for $\operatorname{Re}_{h}=10$, there is a short interval of positive $c$ values with negative $k_{i}$. The corresponding behavior has never been reported for large waves on a falling film (propagating in the positive $x$ direction with the current choice of axes), which suggests that the parameter range where it occurs is not encountered in that context.

As $|c|$ increases further, $k_{r}$ first grows until it reaches a maximum which is visible in Figs. 2(a) and 2(c) for $\Sigma=200$ and 400 . At this maximum the group velocity again diverges and is smaller than the phase velocity between the maximum and the following minimum. With a group velocity smaller than the phase velocity, capillary waves cannot exist ahead of the main wave, which suggests that, again, this parameter range is not realizable for a falling film with developed large waves. Near the minimum the results have a complicated structure shown for $\Sigma=200$ in Figs. 4(a) and 4(b) for $\mathrm{Re}_{h}=0$ and 10, respectively. For $\mathrm{Re}_{h}=0$ the minimum of $k_{r}$ is exceedingly close to zero numerically, but proof that it actually vanishes would require more work. For $\operatorname{Re}_{h}=10$ the minimum of $k_{r}$ is greater than zero and has a finite curvature. In correspondence with this point, $k_{i}$ has a very steep tangent (possibly vertical), as can be seen from the solid lines (red) in Figs. 2(b) and 2(d). In this neighborhood there are three points where $\partial k_{r} / \partial c=0$, which are also points where the group velocity diverges.

\section{COMPARISON}

We are now in a position to prove that the small-amplitude ripples that accompany the large waves in a falling film are essentially linear capillary waves, the wavelength and spatial attenuation of which are determined by the requirement that their phase velocity match that of the primary large wave as in all the wave-hierarchy examples cited in the Introduction and discussed in detail in the references given there. For this purpose we consider nine examples of waves on a falling film and compare the wavelength and rate of spatial attenuation of the capillary waves that accompany the primary large wave with the predictions of the theory of the previous section.

Six of the wave forms are shown in Figs. 5 and 6 with the pertinent parameter values given in Table I. The three examples of Fig. 5 are all for a vertical plate with $\mathrm{Re}=16.1$. The length of the 


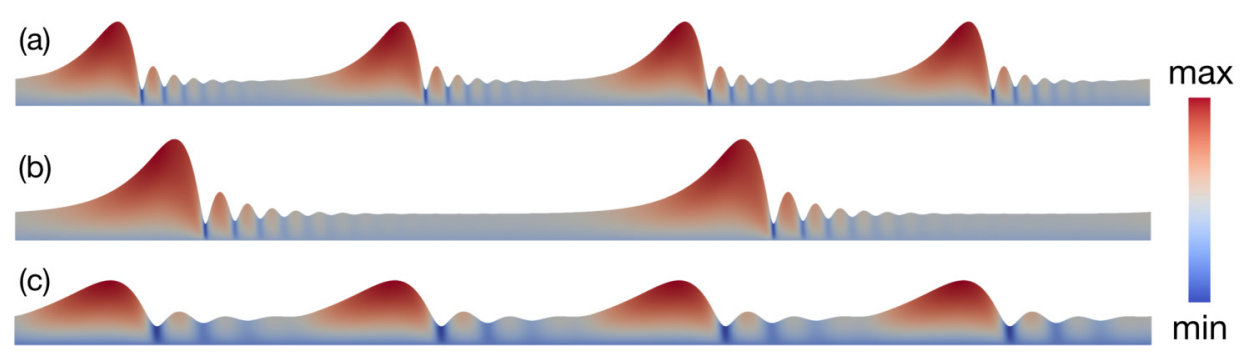

FIG. 5. Computed shapes of some of the waves used for the comparisons of Fig. 7; the pertinent parameters are given in the first three lines of Table I. The sections shown, with a length equal to 460 times the mean film thickness $\bar{h}$, have been obtained by splicing together repeated single waves. The color indicates the velocity parallel to the plate normalized by the flat-film mean velocity (2); the range for each case is specified in Table I. The vertical scale is magnified by a factor of 15 with respect to the horizontal scale.

section shown in the figure is 460 times the mean film thickness $\bar{h}$. In the first example of Fig. 6 , the plate is inclined by $45^{\circ}$, in the second one the film is "hanging" on the underside of a plate inclined by $45^{\circ}$, and in the last one the plate is vertical. For all three of these cases the length of the section shown is $230 \bar{h}$.

In addition to these six cases, we also use three cases from the literature, the parameters of which are given in Table II. The first one is from Fig. 19(b) of Ref. [33], the second one from Fig. 9(d) of Ref. [17], and the last one from Fig. 3 of Ref. [37], as reproduced in Fig. 6.4(a) of Ref. [13]. The first case is the same one used for validation of our method in Fig. 1, while the second case was used for validation in an earlier paper Ref. [24]. For all these cases we performed our own simulations to facilitate the extraction of the wavelength and attenuation of the capillary waves.

In order to proceed with this plan, we need to extract from the results of the simulations the wave number $k_{r}$ and rate of attenuation $k_{i}$ of the capillary waves and the thickness $h$ of the underlying film (which, of course, is different from the mean thickness $\bar{h}$ of the entire liquid flow). The wavelength can be simply determined by measuring the distance between consecutive maxima and minima and averaging. These distances are not precisely equal, but the standard deviation typically does not amount to more than $10 \%$. For the other two quantities the situation is different. The rate of attenuation can be affected by nonlinear effects, and the base film thickness slowly rises towards

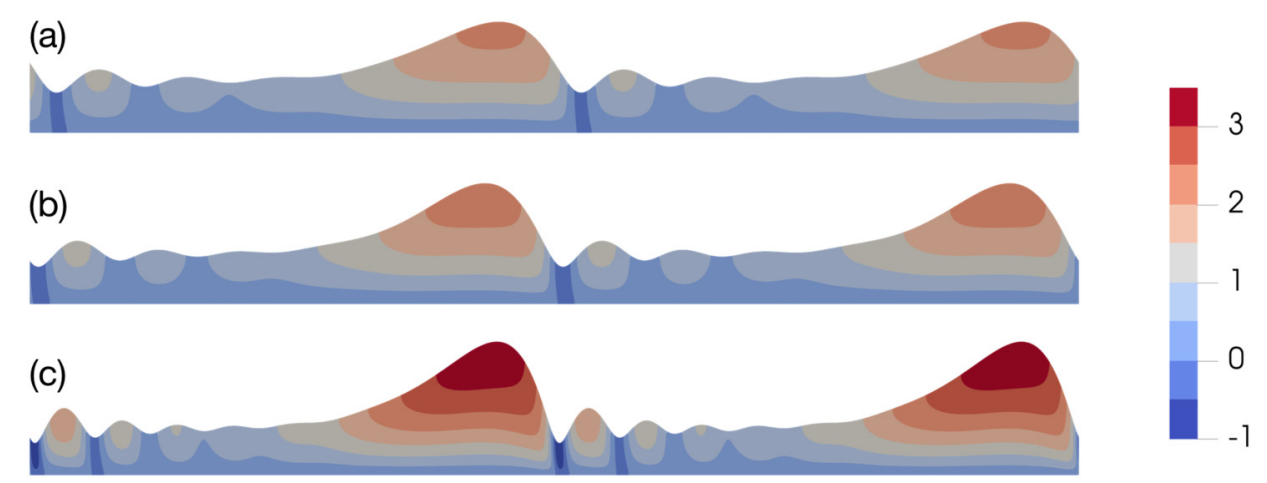

FIG. 6. Computed shapes of some of the waves used for the comparisons of Fig. 7; the pertinent parameters are given in the last three lines of Table I. The length of the sections shown equals to 230 times the mean film thickness $\bar{h}$. The color indicates the velocity parallel to the plate normalized by the flat-film mean velocity for the vertical case; the range for each case is specified in Table I. The vertical scale is magnified by a factor of 15 with respect to the horizontal scale. 
TABLE I. Parameter values for the waves shown in Figs. 5 and 6. The last two columns give the maximum and minimum values of the velocity parallel to the plate, used for the color scale. The flat-film mean velocity (2) is used in the normalization of the first three. For Figs. 6(a) and 6(b), normalization is with the mean velocity for case 6(c).

\begin{tabular}{lcccccc}
\hline \hline Figure & $\mathrm{Re}$ & $\mathrm{Ka}$ & $\ell / \bar{h}$ & $\theta$ & $\max u / \bar{U}$ & $\min u / \bar{U}$ \\
\hline $5(\mathrm{a})$ & 16.1 & 1688 & 115 & $90^{\circ}$ & 3.68 & -1.71 \\
$5(\mathrm{~b})$ & 16.1 & 3376 & 230 & $90^{\circ}$ & 4.53 & -2.30 \\
$5(\mathrm{c})$ & 16.1 & 6752 & 115 & $90^{\circ}$ & 3.13 & -0.68 \\
$6(\mathrm{a})$ & 11.4 & 3789 & 115 & $45^{\circ}$ & 2.13 & -0.19 \\
$6(\mathrm{~b})$ & 11.4 & 3789 & 115 & $-45^{\circ}$ & 2.31 & -0.40 \\
$6(\mathrm{c})$ & 16.1 & 3376 & 115 & $90^{\circ}$ & 3.40 & -1.06 \\
\hline \hline
\end{tabular}

the back of the wave ahead of it (i.e., to the right in the figures). To mitigate the effect of these difficulties, we use the fact that the surface of a flat film with a perfect linear capillary wave of amplitude $A$ at $x=0$ would have the form

$$
\eta=h+A e^{-k_{i} x} \cos \left(k_{r} x\right)
$$

The values of $\eta$ at the maxima and minima of the capillary waves can be used to determine the quantity of interest from a least-squares fit to the computed results. In this calculation we make sure to include an equal number of maxima and minima to achieve a better balance, and we exercise some judgment to try to eliminate the effect of the rising film. This is fairly straightforward when the large waves are broadly spaced and there are numerous capillary waves, such as in the examples of Figs. 5(a), 5(b), and 6(b), while it is less precise when the capillary waves are few and the large waves relatively close to each other as in the other examples in the figures and some of the other cases that we use for comparison. The results of the least-squares fit, the number of capillary waves used in each case, and other pertinent information are shown in Table III.

The results of this procedure are compared with the theory of the previous section in Fig. 7, in which the quantities measured from the simulations, shown on the horizontal axes, are compared with the predicted ones from the previous section on the vertical axes. The left panel is for the wave number $k_{r}$ while the right panel is for the rate of spatial attenuation $k_{i}$, nondimensionalized according to

$$
k_{r}^{*}=\frac{k_{r}}{k_{0}}, \quad k_{i}^{*}=\frac{k_{i}}{2 v k_{0}^{2} / c_{g}^{0}},
$$

with $c_{g}^{0}=\frac{3}{2} c$. Here $k_{0}=\rho c^{2} / \sigma$ is the wave number of inviscid capillary waves having the same phase velocity $c$ as the calculated main wave; $2 v k_{0}^{2}$ is the temporal damping rate of surface waves in the limit of small damping; and division by $c_{g}^{0}$ turns it into a spatial damping rate according to the relation of Gaster [39] [see (A7) in the Appendix]. For both the wave number and the rate of spatial attenuation, the comparison is quite good for most cases, with a maximum difference that does not exceed 5\%. For two points (case (a) of Fig. 5 and the data point from [37]) the difference

TABLE II. Parameter values for the last three cases used for comparison in Fig. 7.

\begin{tabular}{lccccc}
\hline \hline Reference & $\mathrm{Re}$ & $\mathrm{Ka}$ & $\ell / \bar{h}$ & $\theta$ & Symbol \\
\hline$[33]$ & 11.1 & 509.5 & 68.87 & $90^{\circ}$ & Circle \\
{$[17]$} & 12.4 & 346 & 51.88 & $20^{\circ}$ & Diamond \\
{$[37]$} & 19.3 & 526 & 149 & $6.4^{\circ}$ & Cross \\
\hline \hline
\end{tabular}


TABLE III. The first five lines of the table show the results of the least-squares fits of the capillary waves data from Figs. 5, 6, and the three cases from the literature shown in Table II; $h$ is the substrate thickness in the capillary wave region, $\bar{h}$ the mean film thickness for the falling film simulation from Tables I and II, $A$ the amplitude of the capillary wave at the position of the first trough at the foot of the main wave, $k_{r}^{*}$ and $k_{i}^{*}$ the real and imaginary parts of the wave number nondimensionalized as shown in (11), and $N_{\text {waves }}$ the number of waves used for the fit (each one of which provides one value for a maximum and one for a minimum). The last three lines of the table show the values of the dimensionless parameters used in the evaluation of the dispersion relation (8) for each case.

\begin{tabular}{lccccccccc}
\hline \hline & $5(\mathrm{a})$ & $5(\mathrm{~b})$ & $5(\mathrm{c})$ & $6(\mathrm{a})$ & $6(\mathrm{~b})$ & $6(\mathrm{c})$ & Ref. [33] & Ref. [17] & Ref. [37] \\
\hline$h / \bar{h}$ & 0.692 & 0.739 & 0.708 & 0.767 & 0.739 & 0.690 & 0.695 & 0.718 & 0.847 \\
$A / h$ & 0.624 & 0.740 & 0.354 & 0.282 & 0.335 & 0.473 & 0.458 & 0.312 & 0.347 \\
$k_{r}^{*}$ & 1.54 & 1.71 & 3.31 & 2.94 & 3.04 & 2.24 & 1.22 & 0.950 & 0.615 \\
$k_{i}^{*}$ & 15.3 & 21.7 & 167 & 109 & 114 & 46.9 & 6.09 & 2.58 & 1.36 \\
$N_{\text {waves }}$ & 3 & 5 & 2 & 2 & 2 & 3 & 2 & 2 & 3 \\
$h c / v$ & 37.8 & 49.0 & 35.6 & 28.4 & 28.1 & 36.1 & 25.1 & 25.0 & 50.4 \\
$\operatorname{Re}_{h}$ & 5.34 & 6.50 & 5.71 & 5.14 & 4.60 & 5.29 & 3.73 & 4.60 & 11.7 \\
$\Sigma$ & 4254 & 9087 & 17401 & 9431 & 9088 & 8482 & 1139 & 850 & 1719 \\
\hline \hline
\end{tabular}

in the values of $k_{i}^{*}$ is somewhat larger, about $10 \%$, and for the point from Ref. [17] it reaches $30 \%$. A probable cause is a non-negligible effect of the rise of the film thickness trailing the preceding wave.

It is well known in problems of this type that the main wave injects energy into the subsidiary waves that needs to be removed in order for the subsidiary waves to maintain a constant amplitude (see, e.g., Refs. [1,2]). Thus, in the case of a horizontal stream disturbed by a submerged obstacle, for example, gravity waves form downstream of the obstacle so that their energy, that moves slower than the stream, can be carried away by the stream, while capillary waves, whose group velocity is faster than the stream, appear upstream of the obstacle and radiate their energy upstream (see, e.g., Ref. [2]). For the same reason, the parasitic capillary waves at the crest of a nearly breaking gravity wave appear on the forward face of the wave $[8,9]$. This argument, adapted to the present
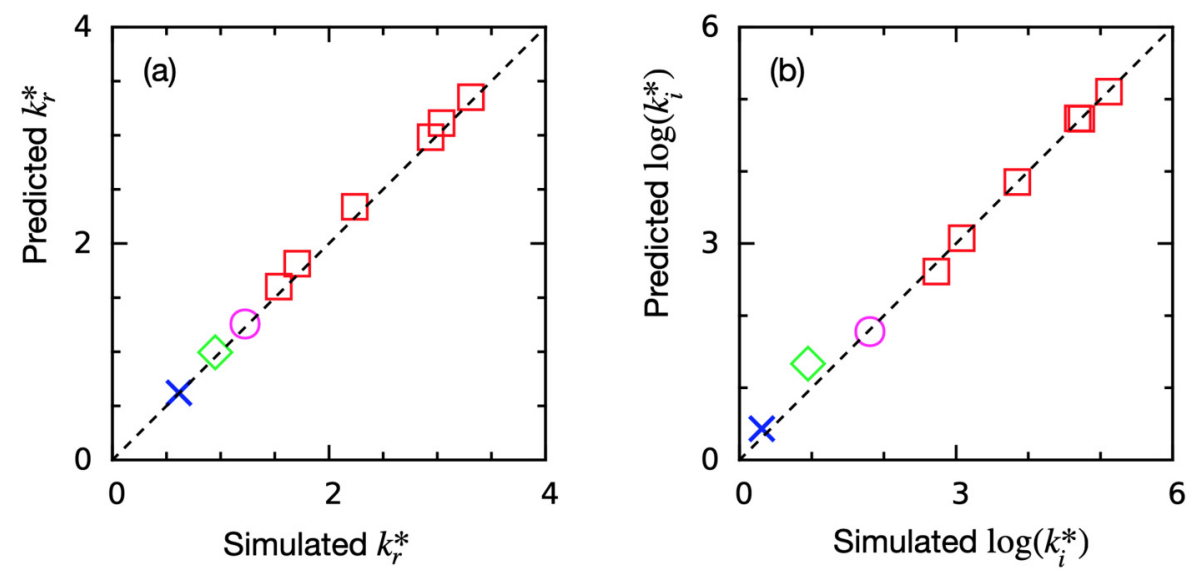

FIG. 7. Comparison between the predicted and calculated values of the wave number (left) and rate of spatial attenuation of the capillary ripples accompanying the large waves shown in Figs. 5 and 6 (squares) and those characterized by the parameters shown in Table II; the cases denoted by circle, diamond, and cross are identified in the table. The nondimensionalizations used are given in (11). 
case of a falling film, shows that if the group velocity of the capillary waves is larger than that of the main wave (which we have verified to be true for all the cases that we have considered), the capillary waves must be located ahead of the main wave so that their energy can be radiated away. Due to the periodicity of the wave system, this energy will be partly dissipated by viscosity and partly radiated toward the wave just ahead, which may provide another mechanism for wave-wave interactions such as those described in Refs. [11,15,17,20].

The Supplemental Material [38] shows the results of a "thought experiment" in which the large wave is allowed to grow in the absence of surface tension effects. When the wave has developed, surface tension is abruptly restored. Still frames and a movie show that a capillary wave starts to appear shortly after this instant and additional ones are generated one after the other. These results suggest that not only the dynamics but the very formation of the small waves are entirely dependent on the action of surface tension and its interaction with inertia and viscosity.

\section{CONCLUSIONS}

We have shown that the wavelength and rate of spatial attenuation of the ripples that accompany the large waves on a falling liquid film are precisely determined by the requirement that they be stationary relative to the main waves. In this sense, the structure of this wave system is similar to the so-called wave hierarchies encountered in many of branches of science. Our study has been based on the linear theory of viscocapillary waves on a falling film. The wave forms shown in Figs. 5 and 6 suggest that some nonlinear effects are probably present in these and, more generally, in other cases as well. However, our results prove that a linear framework is sufficient to elucidate the fundamental physics of the process.

Proof of the result required a nonstandard approach to the calculation of the dispersion relation. The connection with more usual treatments is established in the Appendix. Here it is also shown that the wavelength of these ripples can be closely approximated by that of viscocapillary waves on a nonflowing horizontal liquid film. The rate of spatial attenuation, however, is not accurately reproduced, as the error can be as large as $30 \%$.

\section{ACKNOWLEDGMENTS}

We are grateful to Dr. Di Yang and Meng Li for their help in the initial part of this study. Research reported in this paper was supported by the Gulf Research Program of the National Academies of Sciences, Engineering, and Medicine under Award No. GRP2000008864. The content is solely the responsibility of the authors and does not necessarily represent the official views of the Gulf Research Program or the National Academies of Sciences, Engineering, and Medicine. The numerical computations were carried out on the Sabine cluster of the University of Houston Research Computing Data Core.

\section{APPENDIX: THE DISPERSION RELATION}

In general, a dispersion relation such as the vanishing of the determinant (8) establishes a relation between a complex $k=k_{r}+i k_{i}$ and a complex $c=c_{r}+i c_{i}$ or

$$
\omega=k c=k_{r} c_{r}-k_{i} c_{i}+i\left(k_{i} c_{r}+k_{r} c_{i}\right)
$$

From this expression we find

$$
\operatorname{Re} \frac{d \omega}{d k}=\operatorname{Re}\left(\frac{\partial \omega}{\partial k_{r}}\right)_{k_{i}}=c_{r}+k_{r} \frac{\partial c_{r}}{\partial k_{r}}-k_{i} \frac{\partial c_{i}}{\partial k_{r}}=c_{r}+k_{r} \frac{\partial c_{r}}{\partial k_{r}}+k_{i} \frac{\partial c_{r}}{\partial k_{i}},
$$

where the last step follows from one of the Cauchy-Riemann relations. It is evident from this relation that, if $k_{r} \neq 0, \operatorname{Re} d \omega / d k$ diverges at points where $\partial k_{r} / \partial c_{r}=0$. 
For the standard temporal stability problem, denoted by $T, k=k_{r}$ is real and $c$ or $\omega$ are complex; we write

$$
k(T)=k_{r}(T), \quad c(T)=c_{r}(T)+i c_{i}(T) .
$$

The dispersion relation (8) can be solved for this problem by prescribing $k_{r}$ and solving for $c(T)$. In this case, the speed of propagation of a wave packet localized in space, i.e., the group velocity, is given by (A2) evaluated for $k_{i}=0$ :

$$
c_{g r}(T)=\left[c_{r}+k_{r} \frac{\partial c_{r}}{\partial k_{r}}\right]_{k_{i}=0} .
$$

For the standard spatial stability problem, denoted by $S, k$, and $c$ are complex while $\omega$ is real:

$$
k(S)=k_{r}(S)+i k_{i}(S), \quad c(S)=c_{r}(S)+i c_{i}(S) .
$$

In Ref. [39], Gaster uses the relations

$$
\omega_{i}(T)=\int_{k_{i}(S)}^{0} \frac{\partial \omega_{i}}{\partial k_{i}} d k_{i}=-\int_{0}^{k_{i}(S)} \frac{\partial \omega_{r}}{\partial k_{r}} d k_{i}, \quad \omega_{r}(S)-\omega_{r}(T)=-\int_{0}^{k_{i}(S)} \frac{\partial \omega_{i}}{\partial k_{r}} d k_{i} .
$$

He then observes that, at least in many stability problems, $\partial \omega_{i} / \partial k_{r}$ is small while $\partial \omega_{r} / \partial k_{r}$ is slowly varying with the results

$$
k_{r}(T)=k_{r}(S), \quad \omega_{r}(T) \simeq \omega_{r}(S), \quad \frac{\omega_{i}(T)}{k_{i}(S)} \simeq-\frac{\partial \omega_{r}}{\partial k_{r}},
$$

in which $\partial \omega_{r} / \partial k_{r}$ can be evaluated at any point between $k_{i}=0$ and $k_{i}=k_{i}(S)$. With the former choice, $\partial \omega_{r} / \partial k_{r}$ becomes equal to $c_{g r}(T)$. The last relation connects the temporal and spatial rates of amplification or damping.

The situation analyzed of Sec. III, denoted by the index $N$, is different from both $T$ and $S$. In this case $c=c_{r}$ is real while $k$ and therefore $\omega$ are complex:

$$
k(N)=k_{r}(N)+i k_{i}(N), \quad c(N)=c_{r}(N) .
$$

Since $c_{i}(N)=0$, the analog of (A6) is, in this case,

$$
c_{i}(T)=\int_{k_{i}(N)}^{0} \frac{\partial c_{i}}{\partial k_{i}} d k_{i}=-\int_{0}^{k_{i}(N)} \frac{\partial c_{r}}{\partial k_{r}} d k_{i}
$$

and

$$
c_{r}(N)-c_{r}(T)=\int_{0}^{k_{i}(N)} \frac{\partial c_{r}}{\partial k_{i}} d k_{i} .
$$

In the absence of information on the integrand appearing in the first relation we can appeal to the mean-value theorem to deduce that

$$
c_{i}(T) \simeq-k_{i}(N)\left(\frac{\partial c_{r}}{\partial k_{r}}\right)^{*}
$$

in which the asterisk denotes evaluation at an intermediate value $0 \leqslant k_{i}^{*} \leqslant k_{i}(N)$. If, similarly to (A4), we define a function

$$
V\left(k_{r}, k_{i}\right)=c_{r}\left(k_{r}, k_{i}\right)+k_{r} \frac{\partial c_{r}}{\partial k_{r}}
$$

we see that $V\left(k_{r}, 0\right)=c_{g r}(T)$ and we may rewrite (A11) as

$$
\omega_{i}(T)=k_{r} c_{i}(T)=-k_{i}(N)\left[V-c_{r}\right]_{i}^{*} .
$$



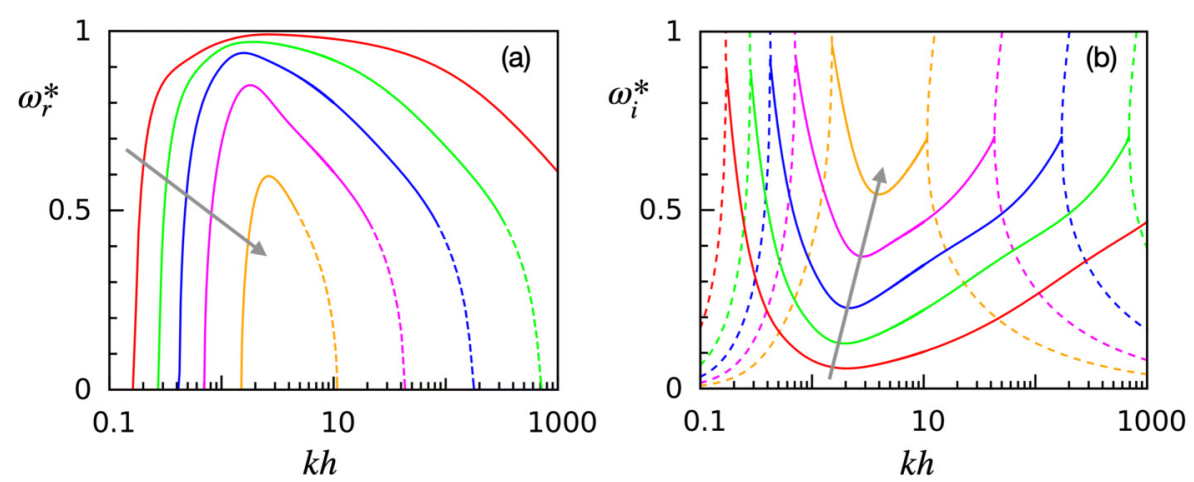

FIG. 8. Real (left) and imaginary parts of the complex frequency $\omega$ normalized as in (A18) of capillary waves on the surface of a viscous liquid layer as functions of $k h$. The curves correspond to different values of the parameter $v / \sqrt{(\sigma / \rho) h}$, which, in the direction of the arrows, takes the values $0.02,0.05,0.1,0.2$, and 0.4. With the nondimensionalization used here, $\omega_{r}^{*}$ equals the phase velocity nondimensionalized by division by the inviscid phase velocity. In the left panel the dashed portion of the lines shows the parameter range in which the group velocity is smaller than the phase velocity. In the right panel the dashed curves are $\omega_{i}^{*}$ in the parameter range where $\omega_{r}=0$.

This relation may be seen as a generalization of (A7) to a situation in which the frame of reference is moving with a velocity $c_{r}$. By determining $\omega_{i}(T)$ from the solution of the dispersion relation (8) for the temporal problem we have verified that, for the cases considered in Sec. IV, the approximation $\left[V-c_{r}\right]_{k_{i}^{*}} \simeq c_{g r}(T)-c_{r}(T)$ is numerically quite good.

Proceeding similarly with (A10) we have

$$
c_{r}(N)=c_{r}(T)+\left(\frac{\partial c_{r}}{\partial k_{i}}\right)^{*} k_{i}(N) .
$$

If $\partial k_{i} / \partial c_{r}$ is large, $c_{r}(N)$ closely approximates $c_{r}(T)$. For each choice of $c_{r}(N)$, the vanishing of the determinant (8) gives a value of $k_{r}$ and, for this value of $k_{r}$, we can solve the $T$ version of the Orr-Sommerfeld equation to find $c_{r}(T)$. We have verified that, for the examples of Sec. IV, the value of $c_{r}(T)$ so determined is numerically very close to $c_{r}(N)$.

If $\operatorname{Re}_{h}=0$ in the characteristic equation (8), aside from the effect of gravity, which is negligible with the small wavelengths of present concern, one finds the dispersion relation for viscocapillary waves on the surface of a liquid layer resting on a horizontal plate, namely,

$$
\begin{aligned}
\Lambda^{4} & +2 \Lambda^{2}+5-\left(\Lambda^{4}+6 \Lambda^{2}+1\right) \frac{\tanh \Lambda k h}{\Lambda} \tanh k h+\left(\tanh k h-\frac{\tanh \Lambda k h}{\Lambda}\right) \Omega_{0}^{2} \\
& =\frac{4\left(\Lambda^{2}+1\right)}{\cosh \Lambda k h \cosh k h}
\end{aligned}
$$

in which

$$
\Lambda=\sqrt{1-\frac{i C}{v k}}, \quad \Omega_{0}^{2}=\frac{\sigma}{\rho v^{2} k} .
$$

In the deep-layer limit $k h \gg 1$, and Eq. (A15) becomes

$$
\left(2-\frac{i C}{v k}\right)^{2}+\frac{\sigma}{\rho \nu^{2} k}=4 \sqrt{1-\frac{i C}{v k}}
$$

which, with $C=\omega / k$, agrees with the standard dispersion relation for viscous capillary waves on deep water (see, e.g., Ref. [40]). If these waves are convected with the surface velocity of the film, $\frac{3}{2} U$, they acquire a phase velocity $c_{r}(T)=C+\frac{3}{2} U$. We have verified that, for the cases of Sec. IV, $c_{r}(N)$ is numerically very close to this quantity. This result suggests that the fact that film is flowing is ultimately of little consequence as far as the phase velocity of the wave is concerned, provided 
that transport at the surface velocity $\frac{3}{2} U$ is accounted for by a simple kinematic transformation. In this sense it may be stated that the ripples accompanying the large waves on a falling film are essentially "ordinary" viscocapillary waves.

In view of its somewhat complicated nature, it may be useful to show some numerical results of the complex frequency predicted by the dispersion relation (A15) vs the wave number $k$ made dimensionless by multiplication by the film thickness $h$. Figure 8 shows the real and imaginary parts of $\omega$ nondimensionalized by the angular frequency of inviscid capillary waves:

$$
\omega^{*}=\omega_{r}^{*}-i \omega_{i}^{*}=\frac{\omega}{\sqrt{(\sigma / \rho) k^{3} \tanh k h}} .
$$

It can be seen in the left panel of the figure that the real part of $\omega$ is nonzero only in a finite range of the parameter $k h$, which becomes larger and larger as $v / \sqrt{(\sigma / \rho) h}$ decreases. For long waves, i.e., small $k h$, the surface perturbation sets into motion the entire liquid layer. The no-slip condition induces a massive amount of dissipation and, as the wavelength increases, the motion changes from damped oscillatory to an aperiodic relaxation. Conversely, for very short waves (large $k h$ ), dissipation is confined to the surface layer, but it rapidly grows with $k$ and, once again, the motion turns from oscillatory to aperiodic as $k h$ increases. The imaginary part of $\omega$ exhibits a minimum in the range where the bottom-induced and surface-induced dissipations are in approximate balance. In the range in which the real part $\omega_{r}$ vanishes, continuation of the complex solution $\pm \omega_{r}-i \omega_{i}$ gives two imaginary branches, both of which are shown as dashed lines.

[1] G. B. Whitham, Linear and Nonlinear Waves (Wiley, New York, 1999).

[2] J. Lighthill, Waves in Fluids (Cambridge University Press, Cambridge, England, 1978), reissued 2001.

[3] E. Raphaël and P.-G. de Gennes, Capillary gravity waves caused by a moving disturbance: Wave resistance, Phys. Rev. E 53, 3448 (1996).

[4] S. Annamalai, S. Balachandar, P. Sridharan, and T. L. Jackson, Pressure evolution equation for the particulate phase in inhomogeneous compressible disperse multiphase flows, Phys. Rev. Fluids 2, 024301 (2017).

[5] M. Watanabe and A. Prosperetti, Shock waves in dilute bubbly liquids, J. Fluid Mech. 274, 349 (1994).

[6] T. Nagatani, The physics of traffic jams, Rep. Prog. Phys. 65, 1331 (2002).

[7] W. Rohlfs and B. Scheid, Phase diagram for the onset of circulating waves and flow reversal in inclined falling films, J. Fluid Mech. 763, 322 (2015).

[8] A. V. Fedorov and W. K. Melville, Nonlinear gravity-capillary waves with forcing and dissipation, J. Fluid Mech. 354, 1 (1998).

[9] M. S. Longuet-Higgins, The generation of capillary waves by steep gravity waves, J. Fluid Mech. 16, 138 (1963).

[10] H.-C. Chang, Wave evolution on a falling film, Annu. Rev. Fluid Mech. 26, 103 (1994).

[11] H.-C. Chang and E. A. Demekhin, Complex Wave Dynamics on Thin Films, (Elsevier, Amsterdam, 2002).

[12] P. L. Kapitza, Wave flow of thin layers of a viscous fluid. I. The free flow, Zh. Eksp. Teor. Fiz. 18, 3 (1948); English translation available in Collected Papers of P. L. Kapitza, edited by D. ter Haar (Pergamon, New York, 1965), Vol. 2, pp. 663-679.

[13] S. Kalliadasis, C. Ruyer-Quil, B. Scheid, and M. G. Velarde, Falling Liquid Films (Springer, New York, 2011).

[14] N. A. Malamataris and V. Balakotaiah, Flow structure underneath the large amplitude waves of a vertically falling film, AIChE J. 54, 1725 (2008).

[15] M. Pradas, S. Kalliadasis, and D. Tseluiko, Binary interactions of solitary pulses in falling liquid films, IMA J. Appl. Math. 77, 408 (2012).

[16] A. Charogiannis, F. Denner, B. G. M. van Wachem, S. Kalliadasis, and C. N. Markides, Detailed hydrodynamic characterization of harmonically excited falling-film flows: A combined experimental and computational study, Phys. Rev. Fluids 2, 014002 (2017). 
[17] F. Denner, A. Charogiannis, M. Pradas, C. N. Markides, B. G. M. van Wachem, and S. Kalliadasis, Solitary waves on falling liquid films in the inertia-dominated regime, J. Fluid Mech. 837, 491 (2018).

[18] D. Gao, N. B. Morley, and V. Dhir, Numerical simulation of wavy falling film flow using VOF method, J. Comput. Phys. 192, 624 (2003).

[19] F. Denner, G. Paré, and S. Zaleski, Dispersion and viscous attenuation of capillary waves with finite amplitude, Eur. Phys. J. Special Topics 226, 1229 (2017).

[20] M. Pradas, D. Tseluiko, and S. Kalliadasis, Rigorous coherent-structure theory for falling liquid films: Viscous dispersion effects on bound-state formation and self-organization, Phys. Fluids 23, 044104 (2011).

[21] T. Kunugi and C. Kino, DNS of falling film structure and heat transfer via MARS method, Comput. Struct. 83, 455 (2005).

[22] A. Samanta, B. Goyeau, and C. Ruyer-Quil, A falling film on a porous medium, J. Fluid Mech. 716, 414 (2013).

[23] G. F. Dietze, On the Kapitza instability and the generation of capillary waves, J. Fluid Mech. 789, 368 (2016).

[24] G. Zhou and A. Prosperetti, A numerical study of mass transfer from laminar liquid films, J. Fluid Mech. 902, A10 (2020).

[25] A. Miyara, Numerical analysis on flow dynamics and heat transfer of falling liquid films with interfacial waves, Heat Mass Transf. 35, 298 (1999).

[26] B. Ramaswamy, S. Chippada, and S. W. Joo, A full-scale numerical study of interfacial instabilities in thin-film flows, J. Fluid Mech. 325, 163 (1996).

[27] T. R. Salamon, R. C. Armstrong, and R. A. Brown, Traveling waves on vertical films: Numerical analysis using the finite element method, Phys. Fluids 6, 2202 (1994).

[28] T. Nosoko and A. Miyara, The evolution and subsequent dynamics of waves on a vertically falling liquid film, Phys. Fluids 16, 1118 (2004).

[29] T. Nosoko, P. N. Yoshimura, T. Nagata, and K. Oyakawa, Characteristics of two-dimensional waves on a falling liquid film, Chem. Eng. Sci. 51, 725 (1996).

[30] P. N. Yoshimura, T. Nosoko, and T. Nagata, Enhancement of mass transfer into a falling laminar liquid film by two-dimensional surface waves - Some experimental observations and modeling, Chem. Eng. Sci. 51, 1231 (1996).

[31] B. Scheid, C. Ruyer-Quil, U. Thiele, O. A. Kabov, J. C. Legros, and P. Colinet, Validity domain of the Benney equation including the Marangoni effect for closed and open flows, J. Fluid Mech. 527, 303 (2005).

[32] D. Yang and L. Shen, Simulation of viscous flows with undulatory boundaries. Part I: Basic solver, J. Comput. Phys. 230, 5488 (2011).

[33] G. F. Dietze, F. Al-Sibai, and R. Kneer, Experimental study of flow separation in laminar falling liquid films, J. Fluid Mech. 637, 73 (2009).

[34] B. E. Anshus and S. L. Goren, A method of getting approximate solutions to the Orr-Sommerfeld equation for flow on a vertical wall, AIChE J. 12, 1004 (1966).

[35] C.-S. Yih, Stability of liquid flow down an inclined plane, Phys. Fluids 6, 321 (1963).

[36] W. H. Press, S. A. Teukolsky, W. T. Vetterling, and B. P. Flannery, Numerical Recipes in Fortran 77 (Cambridge University Press, Cambridge, England, 1992).

[37] J. Liu and J. P. Gollub, Solitary wave dynamics of film flows, Phys. Fluids 6, 1702 (1994).

[38] See Supplemental Material at http://link.aps.org/supplemental/10.1103/PhysRevFluids.5.114005 for a short description and a movie sequence of an "experiment" in which the large wave is allowed to develop without surface tension, which is then abruptly restored. A capillary wave appears shortly thereafter, and subsequent ones are generated one after the other as time elapses.

[39] M. Gaster, A note on the relation between temporally-increasing and spatially-increasing disturbances in hydrodynamic stability, J. Fluid Mech. 14, 222 (1962).

[40] H. Lamb, Hydrodynamics, 6th ed. (Cambridge University Press, Cambridge, England, 1932; reprinted, 1993). 